\title{
Investigation of a Parabolic Iterative Solver for Three-Dimensional Configurations
}

\author{
Douglas M. Nark, Willie R. Watson ${ }^{\dagger}$ and Ramani Mani ${ }^{\ddagger}$
}

\begin{abstract}
parabolic iterative solution procedure is investigated that seeks to extend the parabolic approximation $A$ used within the internal propagation module of the duct noise propagation and radiation code CDUCTLaRC. The governing convected Helmholtz equation is split into a set of coupled equations governing propagation in the 'positive' and 'negative' directions. The proposed method utilizes an iterative procedure to solve the coupled equations in an attempt to account for possible reflections from internal bifurcations, impedance discontinuities, and duct terminations. A geometry consistent with the NASA Langley Curved Duct Test Rig is considered and the effects of acoustic treatment and non-anechoic termination are included. Two numerical implementations are studied and preliminary results indicate that improved accuracy in predicted amplitude and phase can be obtained for modes at a cut-off ratio of 1.7. Further predictions for modes at a cut-off ratio of 1.1 show improvement in predicted phase at the expense of increased amplitude error. Possible methods of improvement are suggested based on analytic and numerical analysis. It is hoped that coupling the parabolic iterative approach with less efficient, high fidelity finite element approaches will ultimately provide the capability to perform efficient, higher fidelity acoustic calculations within complex 3-D geometries for impedance eduction and noise propagation and radiation predictions.
\end{abstract}

\section{Nomenclature}

$\begin{array}{ll}A, B, C & \text { governing equation coupling coefficients } \\ C_{1}, C_{2} & \text { arbitrary integration constants } \\ c & \text { sound speed, } \mathrm{m} / \mathrm{s} \\ F & \text { arbitrary integration constant } \\ i & \sqrt{-1} \\ K & \text { splitting matrix coefficients, } 1 / \mathrm{m} \\ k, k_{x} & \text { free space and axial wave number, } 1 / \mathrm{m} \\ L & \text { duct length, } \mathrm{m} \\ M & \text { uniform steady duct flow Mach number } \\ \mathrm{m}, \mathrm{n} & \text { mode numbers in the } y \text { and } z \text { directions, respectively } \\ Q(x) & \text { arbitrary forcing function }(i . e ., \text { source term) } \\ T(x) & \text { arbitrary splitting matrix (2x2) } \\ x, y, z & \text { axial, horizontal, and vertical duct dimensions, respectively, } \mathrm{m}\end{array}$

Symbols:

$\begin{array}{ll}\alpha, \gamma & \text { complex modal eigenvalues in } y \text { and } z \text { directions, respectively, } 1 / \mathrm{m} \\ \zeta & \text { acoustic impedance, normalized by } \rho c \\ \rho & \text { ambient density, } \mathrm{kg} / \mathrm{m}^{3} \\ \Phi & \text { acoustic potential, } \mathrm{m}^{2} / \mathrm{s} \\ \phi & \text { predicted acoustic potential, } \mathrm{m}^{2} / \mathrm{s}\end{array}$

\footnotetext{
*Research Scientist, Structural Acoustics Branch, Research \& Technology Directorate, AIAA Member

$\dagger$ Senior Research Scientist, Computational Aerosciences Branch, Research \& Technology Directorate, AIAA Senior Member

*Aeroacoustics, LLC
} 
Subscripts:

$\begin{array}{ll}\text { exit } & \text { duct termination } \\ n & \text { iteration level } \\ s & \text { duct inflow (source) }\end{array}$

Superscripts:

$+,-$

'positive' and 'negative' split variable, respectively

\section{Introduction}

$\mathrm{T}$

HE pursuit of accurate and efficient approaches to predict acoustic propagation within complex three-dimensional (3-D) engine nacelles is an important aspect of low-noise engine design studies. Current codes utilize approaches ranging from low fidelity, generally efficient approximate methodologies 12 to high fidelity, computationally intensive, fully elliptic formulations $\frac{3,45}{46,7}$ This investigation focuses on the enhancement of the parabolic approximation used within the internal propagation module of the duct noise propagation and radiation code CDUCT-LaRC ${ }^{8}$ This formulation, originally developed by Dougherty, ${ }^{1}$ provides the capability to study complex, fully three-dimensional configurations in a computationally efficient manner. However, this efficiency comes at the expense of solution fidelity. First, the approach is susceptible to phase errors when acoustic modes near cut-off (i.e., large propagation angles) are considered. Second, the current implementation neglects backward scattering, thus leading to loss of accuracy as reflections from internal bifurcations, liner treatment discontinuities, or duct terminations become important. To alleviate these difficulties, a parabolic iterative approach is investigated that potentially offers improved fidelity over the original formulation while maintaining computational efficiency. In addition, it is hoped that the parabolic iterative solution may be used to accelerate the convergence of iterative, fully elliptic solvers used in higher fidelity methodologies. This study is initiated using a rectangular duct geometry for which acoustic treatment may be included on opposing walls. Paralleling previous studies on the relative fidelity and accuracy of current internal propagation codes, 9 a configuration (see figure 1 ) modeling the Curved Duct Test Rig (CDTR) at NASA Langley ${ }^{10}$ was chosen for initial calculations. An additional reason for considering this geometry is the hope that the proposed iterative technique may be used to accelerate impedance eduction calculations in the CDTR. In the curved duct configuration, three-dimensional impedance eduction will eventually be required.

The following presentation of this work begins with the development of the governing equations in section II An analytical approach is then pursued in section III to identify some possible issues that may arise in the numerical implementation of the proposed parabolic iterative method. This is followed by discussion of the corresponding finite element implementation in section IV. Results for some preliminary calculations are then presented in section $\mathrm{V}$ Finally, some observations and areas for future investigation are discussed in section $\mathrm{VI}$.

\section{Governing Equations}

G OR simplicity, a rectangular duct is considered in which a uniform axial mean flow of Mach number, $M$, is present. Thus, the initial governing equation of the analysis is the convected Helmholtz equation

$$
-\left(1-M^{2}\right) \frac{\partial \Phi}{\partial x^{2}}=\nabla_{\perp}^{2} \Phi-2 i k M \frac{\partial \Phi}{\partial x}+k^{2} \Phi
$$

where $\nabla_{\perp}$ is the transverse Laplacian defined as

$$
\nabla_{\perp}^{2}=\nabla_{\perp} \bullet \nabla_{\perp}=\frac{\partial^{2}}{\partial y^{2}}+\frac{\partial^{2}}{\partial z^{2}}
$$

and an $e^{i \omega t}$ time dependence has been assumed. As discussed by Dougherty 11 and outlined by Corones, 11 a derivation based on splitting the solution of the governing equation (Eq. 1) into 'positive' and 'negative' traveling waves is proposed. This general splitting may be represented as

$$
\begin{gathered}
\left\{\begin{array}{c}
\phi^{+} \\
\phi^{-}
\end{array}\right\}=T(x)\left\{\begin{array}{c}
\Phi \\
\frac{\partial \Phi}{\partial x}
\end{array}\right\} \text { or }\left\{\begin{array}{c}
\Phi \\
\frac{\partial \Phi}{\partial x}
\end{array}\right\}=T(x)^{-1}\left\{\begin{array}{c}
\phi^{+} \\
\phi^{-}
\end{array}\right\} \\
2 \text { of } 16
\end{gathered}
$$


where the splitting matrix, $T(x)$, is arbitrary. As an example, plane waves may be considered to produce a splitting of the form

$$
\left\{\begin{array}{c}
\Phi \\
\frac{\partial \Phi}{\partial x}
\end{array}\right\}=\left[\begin{array}{cc}
1 & 1 \\
\frac{-i k}{1+M} & \frac{i k}{1-M}
\end{array}\right]\left\{\begin{array}{l}
\phi^{+} \\
\phi^{-}
\end{array}\right\}
$$

which was used by Dougherty!1 Substitution of the general variable splitting [Eq. [2]] into the convected Helmholtz equation [Eq. [1]] leads to a coupled pair of differential equations for $\phi^{ \pm}$of the form

$$
\left(1-M^{2}\right) \frac{\partial}{\partial x}\left\{\begin{array}{l}
\phi^{+} \\
\phi^{-}
\end{array}\right\}=\left[\left(1-M^{2}\right)\left(\frac{\partial T}{\partial x}\right) T^{-1}+T\left[\begin{array}{cc}
0 & 1-M^{2} \\
-\left(\nabla_{\perp}^{2}+k^{2}\right) & 2 i k M+M \frac{\partial M}{\partial x}
\end{array}\right] T^{-1}\right]\left\{\begin{array}{l}
\phi^{+} \\
\phi^{-}
\end{array}\right\}
$$

Typically, at this point in the derivation, the terms that couple the equations for $\phi^{ \pm}$are neglected to produce a single parabolic equation for the 'positive' or 'negative' traveling wave. In this work, the coupling terms are maintained and an iterative approach, alternately solving for $\phi^{+}$and $\phi^{-}$, is pursued. To illustrate this more clearly, the simplified case of zero mean flow (i.e., $M=0.0$ ) is considered. The splitting matrix is assumed to be independent of the axial direction, $x$, so that $\frac{\partial T}{\partial x}=0$. However, to allow investigation of the effects of splitting matrix selection on solution accuracy and stability, the splitting matrix is written as

$$
T^{-1}=\left[\begin{array}{cc}
1 & 1 \\
-i K^{+} & i K^{-}
\end{array}\right]
$$

Equation (4) then reduces to the following equations governing $\phi^{ \pm}$

$$
\frac{\partial}{\partial x}\left\{\begin{array}{c}
\phi^{+} \\
\phi^{-}
\end{array}\right\}=\frac{1}{i K^{-}+i K^{+}}\left[\begin{array}{rr}
\left(\nabla_{\perp}^{2}+k^{2}+K^{-} K^{+}\right) & \left(\nabla_{\perp}^{2}+k^{2}-K^{-} K^{-}\right) \\
-\left(\nabla_{\perp}^{2}+k^{2}-K^{+} K^{+}\right) & -\left(\nabla_{\perp}^{2}+k^{2}+K^{-} K^{+}\right)
\end{array}\right]\left\{\begin{array}{l}
\phi^{+} \\
\phi^{-}
\end{array}\right\}
$$

The source boundary condition is given by

$$
\left.\Phi\right|_{x=0}=\phi_{s}
$$

and implementation of the exit impedance boundary condition at the exhaust plane leads to

$$
\left.\frac{\partial \Phi}{\partial x}\right|_{x=L}=\frac{-i k \Phi}{\zeta_{\text {exit }}}
$$

\section{Analytic Approach}

$\mathbf{T}^{\mathrm{N}}$ an effort to gain insight into possible difficulties encountered in iteratively solving Eq. (5) numerically, an analytic approach was pursued. Considering a soft-wall duct mode with eigenvalues $\alpha$ and $\gamma$, and recalling that the axial wave number of the mode is given by $k_{x}^{2}=k^{2}-\alpha^{2}-\gamma^{2}$, Eq. (5) simplifies to

$$
\frac{d}{d x}\left\{\begin{array}{l}
\phi^{+} \\
\phi^{-}
\end{array}\right\}=-i\left[\begin{array}{rr}
A & B \\
C & -A
\end{array}\right]\left\{\begin{array}{l}
\phi^{+} \\
\phi^{-}
\end{array}\right\}
$$

where

$$
A=\frac{k_{x}^{2}+K^{-} K^{+}}{K^{-}+K^{+}}, B=\frac{k_{x}^{2}-K^{-} K^{-}}{K^{-}+K^{+}}, \text {and } C=\frac{-\left(k_{x}^{2}-K^{+} K^{+}\right)}{K^{-}+K^{+}}
$$

The resultant constant coefficient, first order ordinary differential equations may be solved exactly. Writing the equations in the general form $\frac{d g}{d x}+i a g=Q(x)$, the complementary and particular solutions, $g_{c}(x)$ and $g_{p}(x)$ respectively, may be written as

$$
\begin{gathered}
g_{c}=C_{1} e^{-i a x} \\
g_{p}=C_{2} e^{-i a x}+e^{-i a x} \int Q(\xi) e^{i a \xi} d \xi
\end{gathered}
$$

These solutions may then be used to pursue various iterations schemes, computing $\phi_{n}^{ \pm}$at each iteration level to obtain $\phi_{n}=\phi_{n}^{+}+\phi_{n}^{-}$. Two possible approaches are described in subsections $\mathrm{A}$ and $\mathrm{B}$. 


\section{A. Unsymmetric Iteration Scheme}

The first iteration scheme investigated is termed 'unsymmetric', as the application of source terms at the first iteration level is performed in an unsymmetric fashion. $\phi_{0}^{+}$is computed neglecting the $\phi^{-}$source term (i.e., as a homogeneous equation). As the information is available, the resultant solution is used in the source term for $\phi_{0}^{-}$. Equations 9 and (10) lead to solutions on the first level of the form

$$
\begin{aligned}
\phi_{0}^{+} & =F_{0}^{+} e^{-i A x} \\
\phi_{0}^{-} & =F_{0}^{-} e^{i A x}+\frac{C F_{0}^{+}}{2 A} e^{-i A x}
\end{aligned}
$$

where the constants $F_{0}^{ \pm}$are obtained through application of boundary conditions given by Eqs. (6) and (7). For subsequent iteration levels, $n>0$, compute $\phi_{n}^{+}$using the $\phi_{n-1}^{-}$in the source term and $\phi_{n}^{-}$using $\phi_{n}^{+}$in the respective source term. This leads to solutions on the second level of the form

$$
\begin{aligned}
\phi_{1}^{+} & =\left[F_{1}^{+}-\frac{i B C F_{0}^{+}}{2 A} x\right] e^{-i A x}-\left[\frac{B F_{0}^{-}}{2 A}\right] e^{i A x} \\
\phi_{1}^{-} & =\left[F_{1}^{-}+\frac{i B C F_{0}^{-}}{2 A} x\right] e^{i A x}+\left[\frac{C F_{1}^{+}}{2 A}+\left(\frac{C}{2 A}\right)^{2} F_{0}^{+} x\right] e^{-i A x}
\end{aligned}
$$

Note that the first iteration level leads to an expected adjustment of the baseline CDUCT-LaRC solution. However, at this level, terms involving $x$ begin to appear. This is due to the fact that the diagonal terms in the matrix of Eq. (8) are equal, but opposite in sign. Thus, the exponential terms that would normally appear in the integral of Eq. (10) cancel out and lead to 'secular' terms. This level, and subsequent iteration levels, therefore lead to terms that grow axially and are ultimately unstable. Based on these results, it is of interest to include an alternate approach in the investigation to further study the appearance of secular terms.

\section{B. Symmetric Iteration Scheme}

In contrast to the previous approach, the source terms in this iteration scheme are applied in a symmetric fashion. Initially, the source terms for both $\phi_{0}^{+}$and $\phi_{0}^{-}$are neglected, leading to solutions of the form

$$
\begin{aligned}
& \phi_{0}^{+}=F_{0}^{+} e^{-i A x} \\
& \phi_{0}^{-}=F_{0}^{-} e^{i A x}
\end{aligned}
$$

The constants $F_{0}^{ \pm}$are again obtained from Eqs. (6) and 77). For subsequent iteration levels, $n>0$, solutions for $\phi_{n}^{ \pm}$are computed using the previous iteration levels, $\phi_{n-1}^{ \pm}$, in the respective source terms. Following this approach leads to an updated prediction for the first approximation of the form

$$
\begin{aligned}
\phi_{1}^{+} & =F_{1}^{+} e^{-i A x}-\frac{B F_{0}^{-}}{2 A} e^{i A x} \\
\phi_{1}^{-} & =F_{1}^{-} e^{i A x}+\frac{C F_{0}^{+}}{2 A} e^{-i A x}
\end{aligned}
$$

In continuing this approach to the next iteration step, the secular terms that result from the degeneracy of the matrix in Eq. (8) again appear. Thus, while the application of this scheme appears to extend the possible number of iteration levels, it ultimately exhibits the same behavior as the unsymmetric approach of subsection A.

At this point, it is useful to reiterate two observations. First, in looking to accelerate the convergence of fully elliptic solvers used in high fidelity finite element approaches, a minimal number of parabolic iteration steps would be expected. Therefore, in practice, it is hoped that only one or two parabolic iteration steps are required to provide an initial approximation to the finite element approach that results in increased overall efficiency. Secondly, the appearance of the secular terms in both iteration schemes results from the fact that the diagonal terms in the matrix of Eq. (8) are equal, but opposite in sign. Inspection of the more general form of this equation [Eq. (5)] shows this to result from the fact that the transverse Laplacian of the modes describing $\phi_{n}^{ \pm}$are equal. In practice, this will not likely be the case. For example, in a variable area duct or treated duct with flow, the transverse Laplacian of upstream and downstream propagating modes will not be equal. Based on these ideas, it would seem reasonable to follow the initial premise and pursue a finite element implementation of the governing equation that may be easily passed to a fully elliptic finite element approach. 


\section{Numerical Implementation}

THIS section describes the numerical strategy used to obtain the solution to Eq. (5). As mentioned previously, the straight duct was taken to simulate the CDTR (see figure 11). This duct has a horizontal dimension of $0.152 \mathrm{~m}$ (6 in), a vertical dimension of $0.381 \mathrm{~m} \mathrm{(15} \mathrm{in),} \mathrm{and} \mathrm{an} \mathrm{overall} \mathrm{length} \mathrm{of} 0.813 \mathrm{~m} \mathrm{(32} \mathrm{in).} \mathrm{The} \mathrm{chosen} \mathrm{duct}$ has rigid upper and lower walls with acoustic liner treatment on the two sidewalls. A primary reason for choosing the straight CDTR configuration is that its wall lining configuration allows a quasi-3D finite element formulation in the cross-section, while the 'positive' $\left(\phi^{+}\right)$and 'negative' $\left(\phi^{-}\right)$traveling components of the wave field may be obtained at each axial station using a marching method. Here, the decision was made to use a fourth-order Runge-Kutta method to march the wave components along the axis. However, further study of stability limitations will most likely lead to an alternate marching strategy. The presence of acoustic liner treatment on the duct sidewalls is accounted for using a weak form of the wall impedance boundary condition in the finite element formulation. Additionally, the choice of splitting matrices was left arbitrary subject to the assumption of independence on the axial direction. The iterative scheme used in the numerical approach was implemented in a manner that parallels the analytic approach in section III

\section{A. Unsymmetric Iteration Scheme (PIE1)}

The unsymmetric iteration scheme (also referred to as PIE1) progresses as follows.

1. Set the initial value of the predicted acoustic potential, $\phi_{0}^{+}$, to the specified source value

$$
\phi_{0}^{+}(0, y, z)=\phi_{s}(y, z)
$$

Note that, if reflections are present, this boundary condition contains contributions from both the downstream and upstream propagating waves. The equation governing $\phi_{0}^{+}[$Eq. $[5]$ ] is integrated at subsequent axial locations with source terms set to zero to carry the prediction from the source location to the duct termination. This initial step matches the current approach used in the propagation module of CDUCT-LaRC. The following steps are attempts to use the previously described iterative approach to improve accuracy by accounting for backward scattering and, possibly, wide angle propagation.

2. At the duct termination, the initial value of $\phi_{0}^{-}$is obtained by applying the outflow boundary condition, which leads to

$$
\phi_{0}^{-}(L, y, z)=\left(\frac{K^{+}-\frac{k}{\zeta_{\text {exit }}}}{K^{-}+\frac{k}{\zeta_{\text {exit }}}}\right) \phi_{0}^{+}(L, y, z)
$$

Subsequent values at each axial location are determined by integrating the equation governing $\phi_{0}^{-}[\mathrm{Eq} . \quad 5 \mathrm{p}]$ ] and marching from the termination to the source location using $\phi_{0}^{+}$in the source term. Note that at this stage, the resultant combination of the two approximations, $\phi=\phi_{0}^{+}+\phi_{0}^{-}$, satisfies the exit impedance condition [Eq. [7] ]. However, the inflow (i.e., source) condition may not be fully satisfied, as $\phi_{0}^{-}$will in general not be zero at the source location. Typically, the source information is known to a greater degree than the exit impedance and satisfaction of the inflow condition will be of greater interest. Therefore, the first approximation is obtained by one further step.

3. In order to satisfy the inflow condition, the initial value is obtained from

$$
\phi_{1}^{+}(0, y, z)=\phi_{s}(y, z)-\phi_{0}^{-}(0, y, z)
$$

The calculation for $\phi_{1}^{+}$is then carried from the source location to the duct termination using the previous approximation, $\phi_{0}^{-}$, in the source term. Thus, the first approximation is obtained by combining the two previous approximations to obtain

$$
\phi_{1}=\phi_{1}^{+}+\phi_{0}^{-}
$$

This first approximation then satisfies the source condition. Additionally, as $\phi_{1}^{+}$is hopefully a minor correction to $\phi_{0}^{+}$, the outflow boundary condition is nearly satisfied.

4. For subsequent iteration steps (i.e., $n>1$ ), the initial value of $\phi_{n-1}^{-}$at the duct termination is again obtained by applying Eq. 14 using $\phi_{n-1}^{+}$approximation values. The solution is then marched from duct termination to 
source location by solving Eq 5. In order to satisfy the source condition, the initial value of $\phi_{n}^{+}$is obtained using Eq. (6) in the form

$$
\phi_{n}^{+}(0, y, z)=\phi_{s}(y, z)-\phi_{n-1}^{-}(0, y, z)
$$

The calculation for $\phi_{n}^{+}$is then carried from the source location to the duct termination using the previous approximation, $\phi_{n-1}^{-}$, values in the source terms of Eq. 55. The $n^{\text {th }}$ approximation is then obtained by combining the two approximations to obtain

$$
\phi_{n}=\phi_{n}^{+}+\phi_{n-1}^{-}
$$

\section{B. Symmetric Iteration Scheme (PIE2)}

The symmetric iteration scheme (also referred to as PIE2) progresses as follows.

1. Set the initial value of the predicted acoustic potential, $\phi_{0}^{+}$, to the specified source value

$$
\phi_{0}^{+}(0, y, z)=\phi_{s}(y, z)
$$

The equation governing $\phi_{0}^{+}$[Eq. [5]] is integrated at subsequent axial locations with source terms set to zero to carry the prediction from the source location to the duct termination. As with the previous iteration scheme, termination of the solution procedure at this point matches the current approach contained in the propagation module of CDUCT-LaRC. Attempting to improve the accuracy using the symmetric iterative approach proceeds as follows.

2. At the duct termination, the initial value of $\phi_{0}^{-}$is obtained by applying the outflow boundary condition which leads to

$$
\phi_{0}^{-}(L, y, z)=\left(\frac{K^{+}-\frac{k}{\zeta_{\text {exit }}}}{K^{-}+\frac{k}{\zeta_{\text {exit }}}}\right) \phi_{0}^{+}(L, y, z)
$$

At this point, the procedure differs from the unsymmetric scheme. In the current approach, subsequent values at each axial location are determined by integrating the equation governing $\phi_{0}^{-}$[Eq. [5] ] with the source term set to zero and marching from the termination to the source location .

3. Again, as the inflow condition is generally not satisfied, Eq. (6) is used to obtain the initial value

$$
\phi_{1}^{+}(0, y, z)=\phi_{s}(y, z)-\phi_{0}^{-}(0, y, z)
$$

The calculation for $\phi_{1}^{+}$is then carried from the source location to the duct termination using the previous approximation, $\phi_{0}^{-}$, in the source terms. Thus, the first approximation is obtained by combining the two previous approximations to obtain

$$
\phi_{1}=\phi_{1}^{+}+\phi_{0}^{-}
$$

The source condition is then satisfied and the outflow boundary condition is nearly satisfied, as $\phi_{1}^{+}$is hopefully a minor correction to $\phi_{0}^{+}$.

4. For subsequent iteration steps (i.e., $n>1$ ), the procedure proceeds in a fashion similar to the unsymmetric approach. The initial value of $\phi_{n-1}^{-}$at the duct termination is again obtained by applying Eq. 16 using $\phi_{n-1}^{+}$ approximation values. The solution is then marched from duct termination to source location by solving Eq (5). In order to satisfy the source condition, the initial value of $\phi_{n}^{+}$is obtained using Eq. 6 in the form

$$
\phi_{n}^{+}(0, y, z)=\phi_{s}(y, z)-\phi_{n-1}^{-}(0, y, z)
$$

The calculation for $\phi_{n}^{+}$is then carried from the source location to the duct termination using the previous approximation, $\phi_{n-1}^{-}$, values in the source terms of Eq. 5. The $n^{t h}$ approximation is then obtained by combining the two approximations to obtain

$$
\phi_{n}=\phi_{n}^{+}+\phi_{n-1}^{-}
$$

The aforementioned iterative procedures may be continued to desired levels based on various criteria. It should again be noted that in order to guarantee satisfaction of the inflow condition [Eq. [6] ], iterations were terminated at the $\phi_{n}^{+}$ level. This differs slightly from the procedure described in the analytic approach (section III). The knowledge obtained from the analytic solutions should nevertheless carry over to the numerical results. 


\section{Results}

$\mathrm{T}$ HE numerical implementations were applied to various test problems with and without wall treatment, as well as anechoic and non-anechoic terminations. As an example of baseline calculations, the rectangular duct was taken to be hardwall with a plane wave source at a frequency of $2000 \mathrm{~Hz}$. This mode propagates at an angle of zero relative to the duct axis (i.e., is well cut-on), thus alleviating the wide angle propagation issues of the parabolic approximation. With no reflections, it would therefore be expected that the first pass, $\phi_{0}^{+}$, of the parabolic iterative procedure would capture the solution very well. The subsequent second pass, $\phi_{0}^{-}$, should then be zero (or extremely small). The values of $K^{ \pm}$in the splitting matrix were set to the free space wave number of $k$, as in Eq. (3) for no flow. Note that this is the same splitting found in the current propagation module of CDUCT-LaRC. The predicted real part of acoustic pressure using the PIE1 approach is compared with the analytic solution in figure 2. To introduce the format in which further results are discussed, figure 3 is also included. Here, the amplitude error is presented in decibels referenced to the exact solution at the given axial location. The phase error (difference between predicted and exact phase) in degrees is also provided. The red lines (with circles) represent the initial prediction $\left(\phi_{0}^{+}\right)$that corresponds to a current CDUCT-LaRC solution. The green lines (with triangles) represent the first approximation as described in section IV] It can be seen that the first pass captures the solution very well. The resultant zero value for $\phi_{0}^{-}$shows that, as expected, there is little to be gained in trying to obtain a correction.

Previous work ${ }^{912}$ has shown that CDUCT-LaRC handles plane wave cases very well. Therefore, excellent results for plane wave cases (hardwall and treated) are not new or unexpected. However, this case does show that the proposed iterative scheme behaves as expected and remains stable when an accurate first approximation is obtained. This may be further verified by inspection of Eq. (5). It can be seen that as the value of the splitting terms $\left(K^{ \pm}\right)$approach that of the axial wave number $\left(k_{x}\right)$, the coefficients of the coupling terms (matrix diagonals $B, C$ ) become smaller. In this case, the splitting terms were set to $k$. For the plane wave, this is equal to the axial wave number so that the coupling terms are negligible. Also, since there were no reflections (either internal or from the duct termination), the value of $\phi_{0}^{-}$was zero. Further calculations for higher order modes show similar results when splitting matrices are chosen that more closely approximate the true axial wave number. Obviously, this is much more difficult when a general source description is involved. However, although not a focus of this work, it does illustrate that judicious selection of the splitting matrix may offer some possibility for improving accuracy.

\section{A. Hardwall Cases}

With the predictions for the plane wave cases developing as expected, focus turned to higher order mode predictions. Following previous work, 9 the $(0,1),(1,0)$ and $(1,3)$ modes at a source frequency of $2000 \mathrm{~Hz}$ and an anechoic termination were investigated. As the $(0,1)$ mode is well cut-on with a cut-off ratio of 4.46 , initial CDUCT-LaRC predictions matched the analytic solution very well. Subsequent iterations again showed the approach to be stable for good initial approximations and the behavior was similar to that for the plane wave cases. In light of this, figures for these predictions were not included. Consequently, hardwall predictions for the $(1,0)$ mode, which had a cut-off ratio of 1.75 , were conducted. In this instance, the initial CDUCT-LaRC predictions would be expected to show phase error with minimal amplitude error. The results from these initial predictions, as well as subsequent iterations, for both numerical approaches are presented in figures 4 and 5 . The plotting style is the same as that used for the plane wave case. However, these figures also include a blue line (with squares) that represents the second approximation in the iteration schemes. It can be seen that both approaches lead to improvements in the predicted phase. It appears that at least some of this improvement in the phase comes at the expense of amplitude accuracy. However, the amplitude error for the second approximation in the PIE1 approach is quite low. These predictions also show indications of the effects of the secular terms discussed in section III] as the error appears to increase with axial distance.

In addition to the predictions for an anechoic duct termination, cases involving a non-anechoic duct termination were also investigated. Figures 6 and 7 illustrate results for the $(1,0)$ mode under the same conditions as above, except that an arbitrary impedance of $(2.0,0.0)$ was prescribed at the duct termination. The amplitude error plots clearly show evidence of a standing wave pattern, and the second approximations for both approaches show improved amplitude prediction. Additionally, the slight variation in the phase appears to be captured more accurately by the iteration schemes.

\section{B. Treated Cases}

As an extension to the hardwall case and to match the CDTR configuration, acoustic treatment was also incorporated on the side wall of the duct. The normalized impedance of the treatment was set to an arbitrary value of (1.0,-0.5), 
an anechoic termination was assumed, and the source frequency was again set to $2000 \mathrm{~Hz}$. The predictions began with the least attenuated, $n=1$ transverse (hardwall direction) mode and results are presented in figures 8 and 9 For the PIE1 approach in particular, improvement in the predicted amplitude may be seen. The predicted phase for all approximations match quite well. Generally, it has been expected that the inclusion of acoustic treatment will diminish the importance of CDUCT-LaRC wide angle propagation errors, as acoustic modes tend to interact more with duct walls as cut-off is approached. These results indicate that the iterative procedure appears to follow similar trends.

In light of the previous predictions, a non-anechoic duct termination $\left[\zeta_{\text {exit }}=(2.0,0.0)\right]$ was incorporated into the treated duct. The resulting predictions are shown in figures 10 and 11 . Again, the iteration schemes provide a great deal of improvement in the amplitude prediction. Additionally, the iterated phase predictions capture slight variations evident in the exact solution that do not appear in the initial approximations.

With the improvements indicated in previous examples, the predictions were extended to the $(1,3)$ mode to investigate even larger propagation angles. Having a cut-off ratio of 1.13 in a hardwall configuration, the initial CDUCTLaRC predictions would be expected to show much larger phase error, as well as an indication of increased amplitude error. This is due to the fact that the mode propagates at a considerably larger angle than the preferred propagation direction assumed by the parabolic approximation currently utilized in CDUCT-LaRC. Initially, an anechoic termination was considered and the predictions are illustrated in figures 12 and 13 The same non-anechoic termination $\left[\zeta_{e x i t}=(2.0,0.0)\right]$ was also considered and the subsequent predictions are presented in figures 14 and 15 In these results, the indications of secular terms are more evident, as the amplitude errors appear to increase with increasing axial extent. Nevertheless, improvement in the phase predictions is also evident. Again, it also appears that some improvement in predicted phase was obtained at the expense of amplitude error. The implications of this trade-off for these modes are not completely clear. However, as mentioned previously, it is hoped that for cases involving many modes, the increased effectiveness of acoustic treatment on modes near cut-off will offer some further relief.

\section{Concluding Remarks}

$\mathrm{B}$ ASED on preliminary calculations such as those discussed above, improved solution fidelity over the current parabolic approach used in CDUCT-LaRC may be obtained by considering an iterative parabolic approach. This improvement comes at a relatively small increase in computational expense in comparison to wide angle corrections. In particular, improvements in both amplitude and phase were evident in cases involving reflections from the duct termination. It is anticipated that similar improvements will carry over to cases in which backward scattering from features such as bifurcations or impedance discontinuities are non-negligible. Predictions incorporating acoustic treatment also showed similar qualities. Generally, the PIE1 numerical approach appeared to perform better than the PIE2 approach. This may be due to that fact that source terms are included for both $\phi^{ \pm}$at all stages of the PIE1 iteration process. This differs from the PIE2 approach in which source terms are not applied in calculating the initial solution for $\phi_{0}^{-}$. This follows the observations drawn from the analytic approach of section III

As expected, predictions for the (1,3) mode (cut-off ratio of 1.13) showed larger amplitude and phase errors relative to the lower order mode cases. For both implementations, the iterative approach provided improved phase prediction. However, this was accompanied by an increase in the amplitude error. Considering the splitting matrix employed, as well as previous experience, this is not surprising. With the large propagation angle of this mode, larger error in the initial $\phi^{+}$would be expected at the duct termination. This in turn would require large $\phi^{-}$values to satisfy the duct termination boundary condition. Essentially, this situation leads to large 'artificial' reflection factors at the duct termination, producing large coefficients for the coupling terms. In light of this, the investigation shows that a judicious choice of splitting matrix may alleviate a great deal of this error. It may also be possible to reduce this error by considering an alternative termination boundary condition in which the value $\phi_{n}^{-}$is set to zero at each iteration level. This would bound the initial $\phi^{-}$values and possibly provide stability.

Overall, this initial implementation has shown some of the possible benefits of the iterative approach and provided a basis from which further investigation may proceed. It is hoped that continued development of this parabolic iterative approach will ultimately provide the capability to perform efficient, higher fidelity acoustic predictions within complex 3-D geometries. One possible extension would be to utilize high fidelity axi-symmetric (or quasi-3D) solution to initialize the parabolic iterative scheme. Improved splitting matrices derived from the initial solution could provide more accurate 3-D predictions with limited iteration steps. Conversely, the approach might also prove to be useful in accelerating the convergence of iterative, fully elliptic solvers used in the less efficient, high fidelity finite element approaches. 


\section{References}

${ }^{1}$ Dougherty, R. P., "A Parabolic Approximation for Flow Effects on Sound Propagation in Nonuniform, Softwall, Ducts," AIAA Paper 99$1822,1999$.

${ }^{2}$ Lan, J. H., “Turbofan Duct Propagation Model,” NASA CR 2001-211245, 2001.

${ }^{3}$ Watson, W. R., "Three-Dimensional Rectanguar Duct Code with Application to Impedance Eduction," AIAA Journal, Vol. 40, No. 2, 2002, pp. 217-226.

${ }^{4}$ Lan, J. H. and Breard, C., "Development and Validation of a 3D Linearized Euler Solver," AIAA Paper 2006-2585, 2006.

${ }^{5}$ Lan, J. H. and Breard, C., "Validation of 3D Acoustic Propagation Code with Analytical and Experimental Results," AIAA Paper 2005-2901, 2005.

${ }^{6}$ Mosson, A., Caro, S., Knapen, T., Gontier, M., Enault, L., and Drouilhet-Peyre, S., "New advances in the use of Actran/TM for nacelle simulations and optimisation of IBM clusters for Actran parallel computations," AIAA Paper 2006-2588, 2006.

${ }^{7}$ Free Field Technologies, Louvain-la-Neuve, Belgium, MSC.ACTRAN 2005 User's Manual, 2005.

${ }^{8}$ Nark, D. M., Farassat, F., Pope, D. S., and Vatsa, V., "The Development of the Ducted Fan Noise Propagation and Radiation Code CDUCTLaRC," AIAA Paper 2003-3242, 2003.

${ }^{9}$ Nark, D. M. and Farassat, F., "Further Investigation of Acoustic Propagation Codes for Three-Dimensional Geometries," AIAA Paper 20062586, 2006.

${ }^{10}$ C.Gerhold, Cabell, R., and Brown, M., "Development of an Experimental Rig for Investigation of Higher Order Modes in Ducts," AIAA Paper 2006-2637, 2006.

${ }^{11}$ Corones, J., "Bremmer Series that Correct Parabolic Approximations," Journal of Mathematical Analysis and Applications, Vol. 50, 1975, pp. 361-372.

${ }^{12}$ Nark, D. M., Watson, W. R., and Jones, M. G., "An Investigation of Two Acoustic Propagation Codes for Three-Dimensional Geometries," AIAA Paper 2005-3022, 2005.
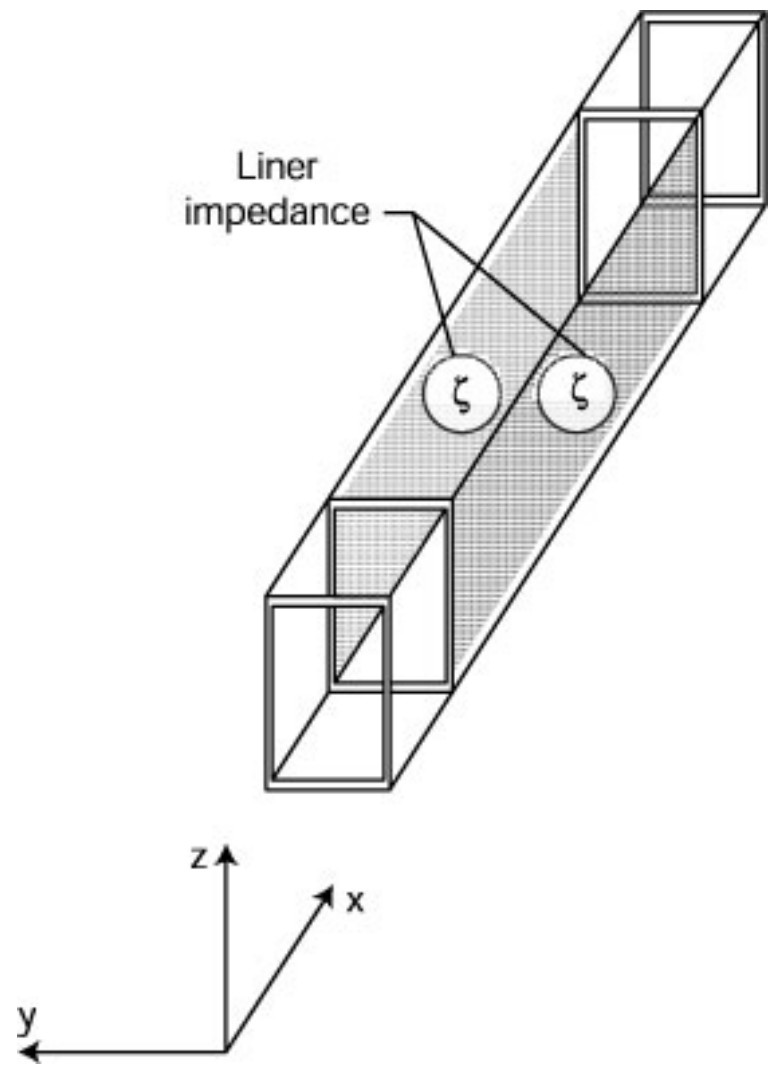

Figure 1. Three dimensional duct and Cartesian coordinate system 


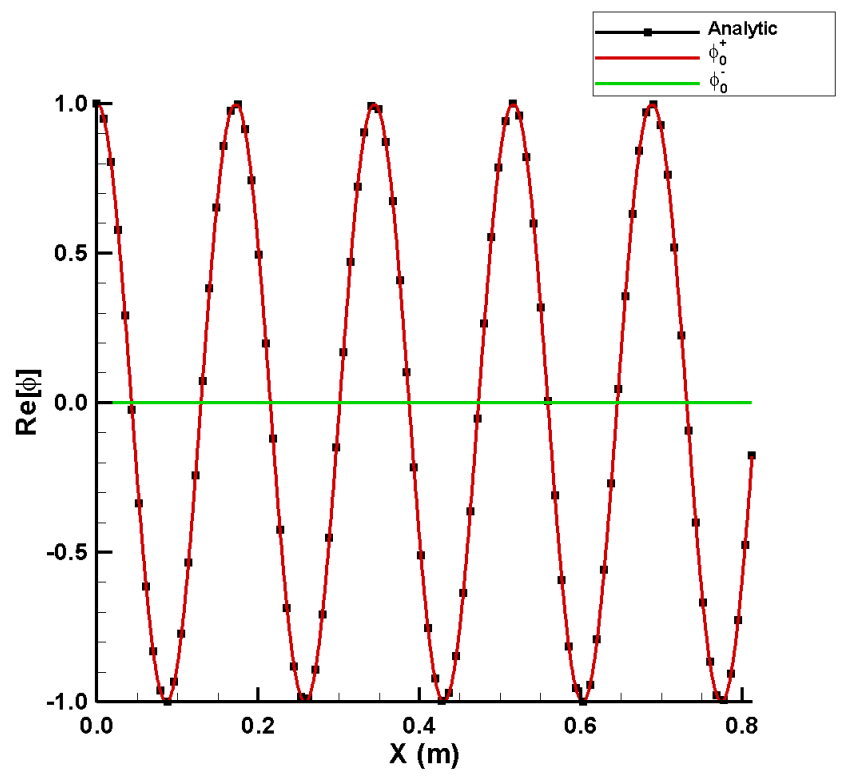

Figure 2. Comparison of predicted real part of acoustic potential with the analytic solution [plane wave, hardwall duct: $f=2000 \mathrm{~Hz}$ ]

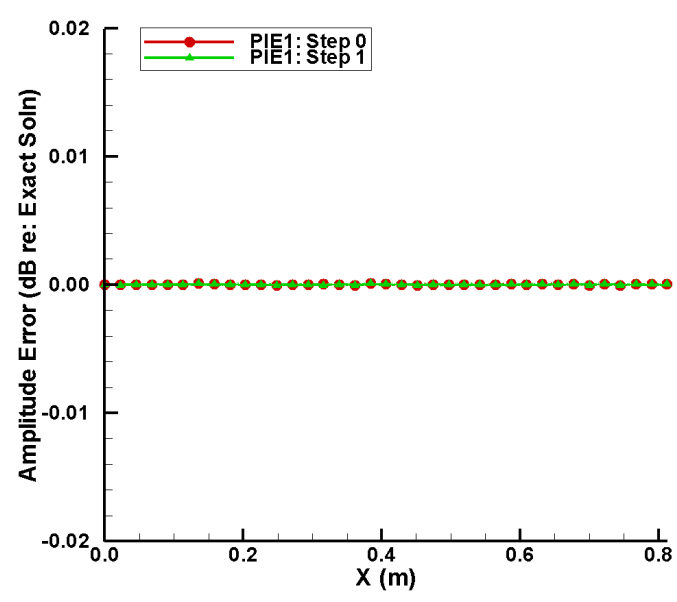

(a) Amplitude Error

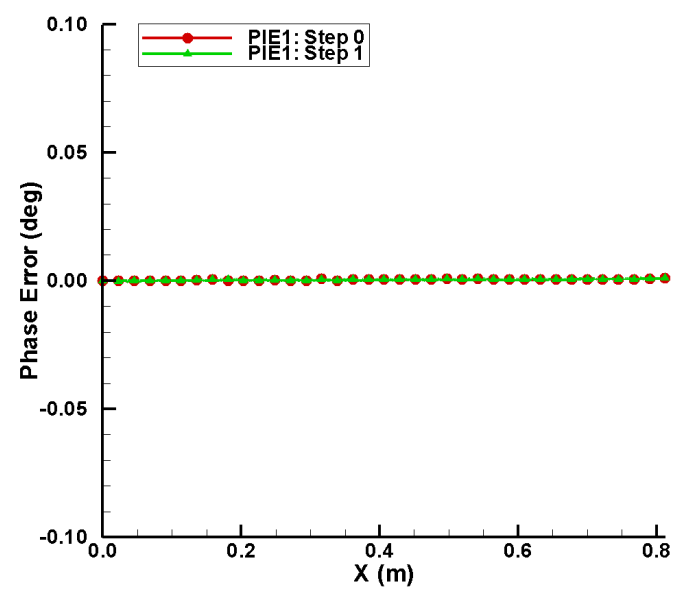

(b) Phase Error

Figure 3. Amplitude and phase error for several PIE1 iteration steps (plane wave, hardwall duct: $f=2000 \mathrm{~Hz}$ ) 


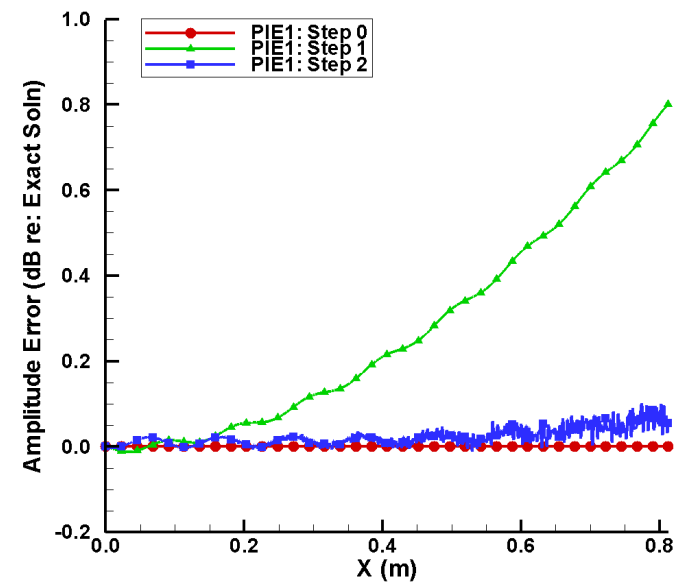

(a) Amplitude Error

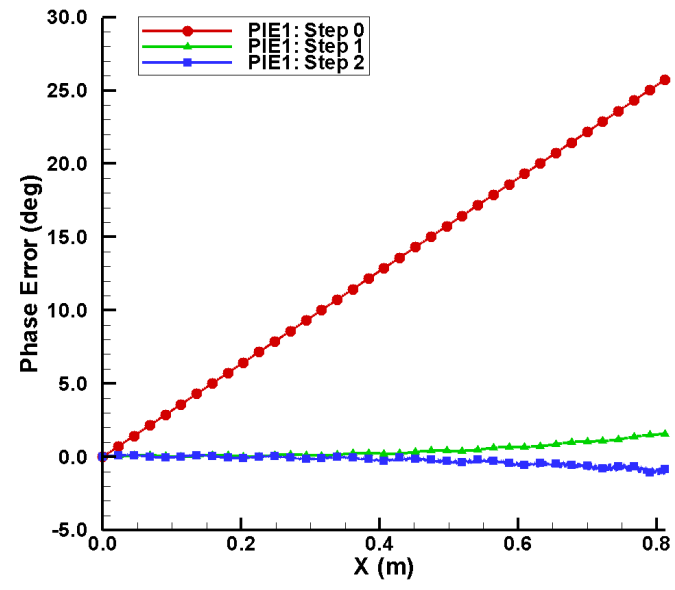

(b) Phase Error

Figure 4. Amplitude and phase error for several PIE1 iteration steps (mode [1,0], hardwall duct: $f=2000 \mathrm{~Hz}$ )

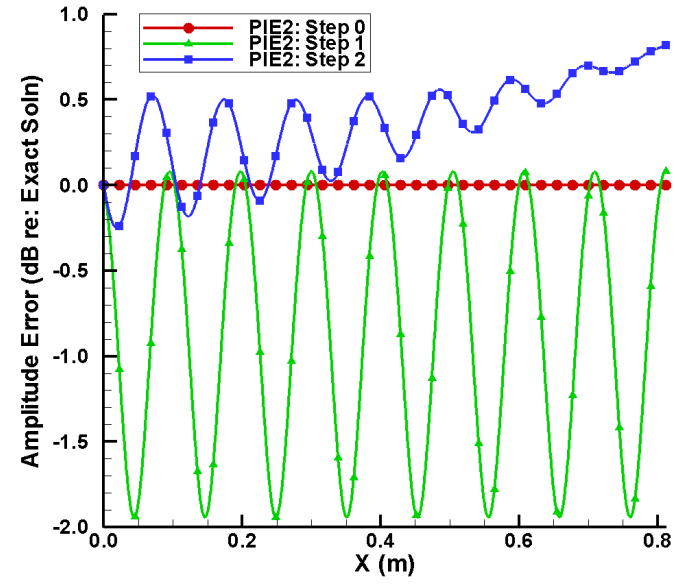

(a) Amplitude Error

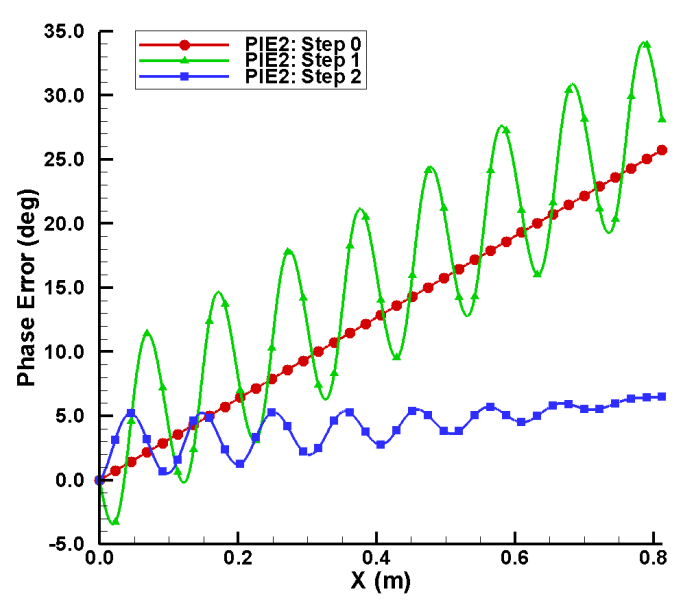

(b) Phase Error

Figure 5. Amplitude and phase error for several PIE2 iteration steps (mode [1,0], hardwall duct: $f=2000 \mathrm{~Hz}$ ) 


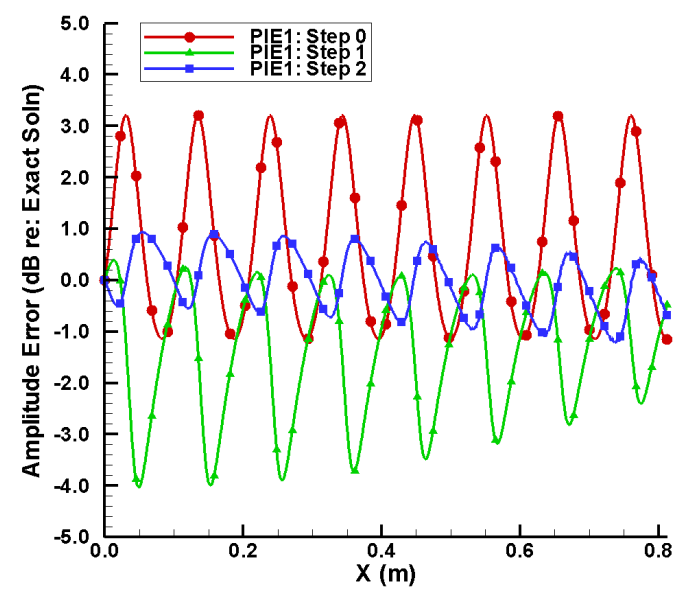

(a) Amplitude Error

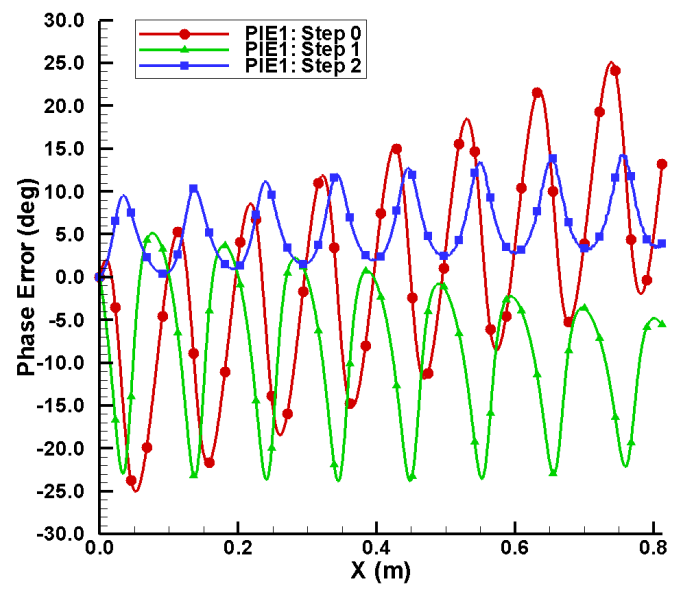

(b) Phase Error

Figure 6. Amplitude and phase error for several PIE1 iteration steps with reflections (mode [1,0], hardwall duct: $\zeta_{e x i t}=(2.0,0.0)$, $f=2000 \mathbf{H z})$

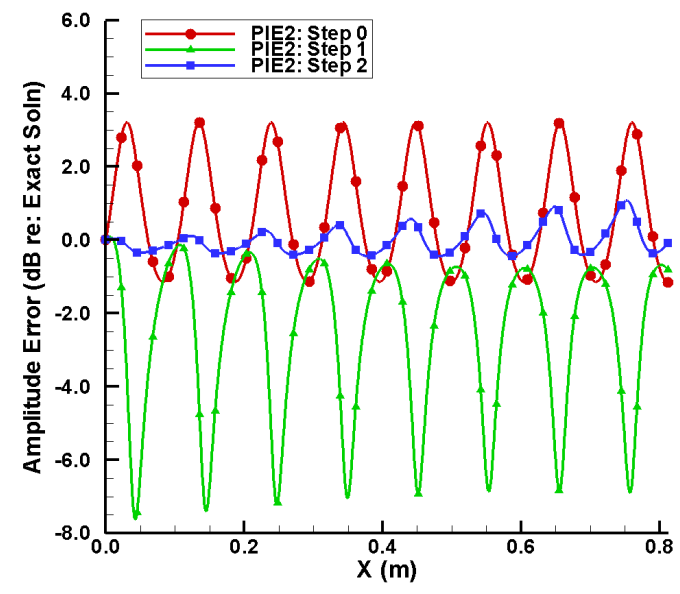

(a) Amplitude Error

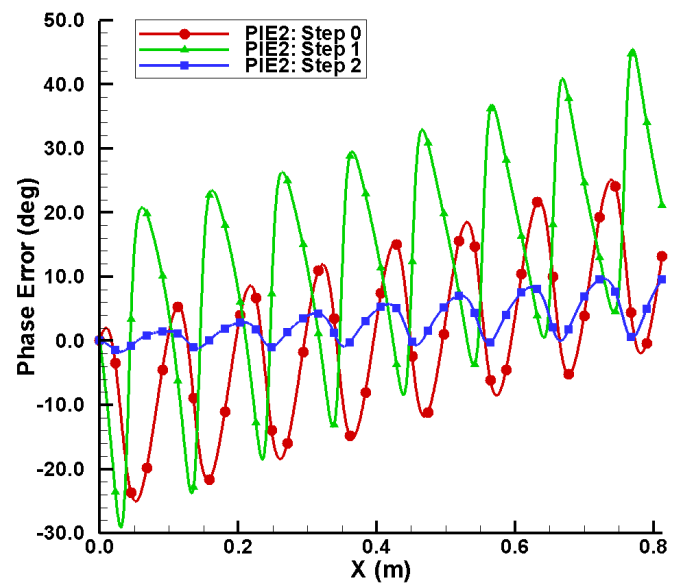

(b) Phase Error

Figure 7. Amplitude and phase error for several PIE2 iteration steps with reflections (mode [1,0], hardwall duct: $\zeta_{e x i t}=(2.0,0.0)$, $f=2000 \mathbf{H z})$ 


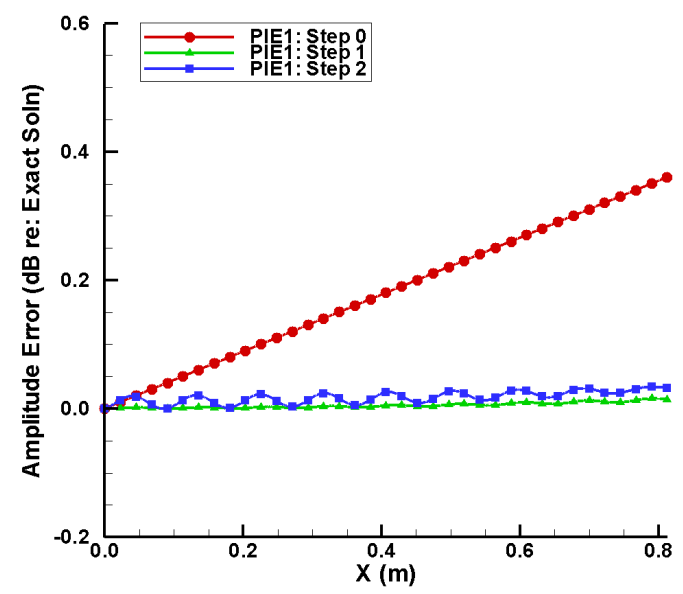

(a) Amplitude Error

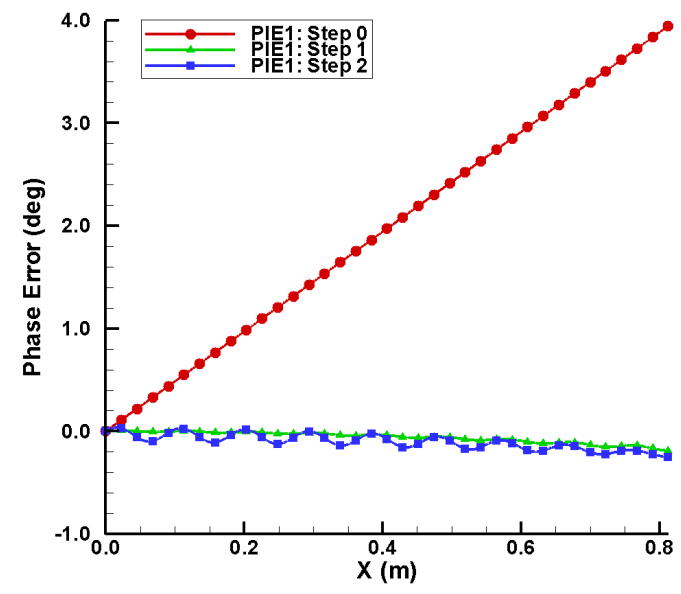

(b) Phase Error

Figure 8. Amplitude and phase error for several PIE1 iteration steps (least attenuated mode, treated duct: $n=1, \zeta=(1.0,-0.5)$, $f=2000 \mathbf{H z})$

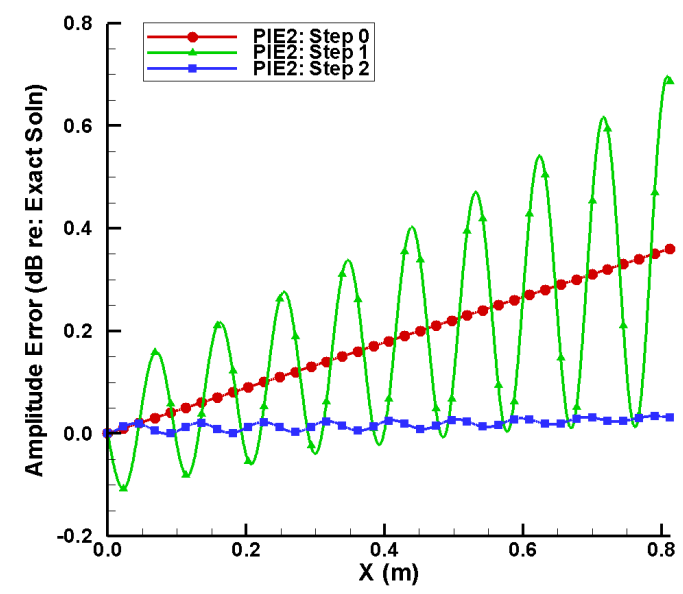

(a) Amplitude Error

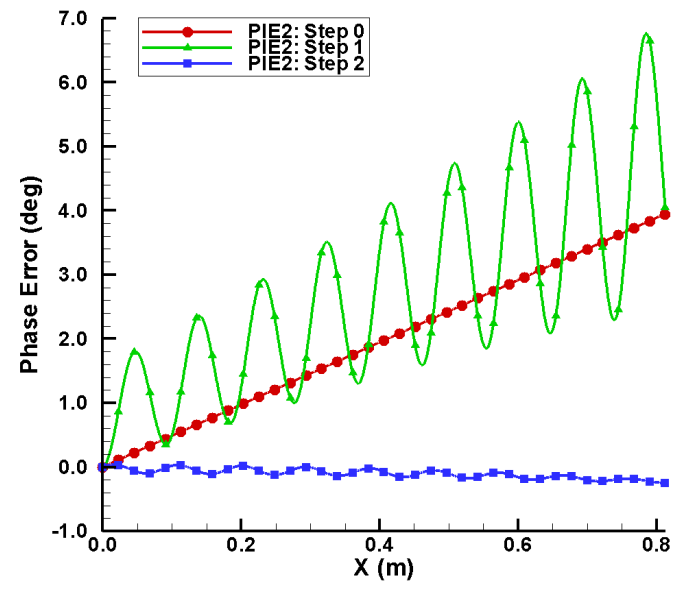

(b) Phase Error

Figure 9. Amplitude and phase error for several PIE2 iteration steps (least attenuated mode, treated duct: $n=1, \zeta=(1.0,-0.5)$, $f=2000 \mathbf{H z})$ 


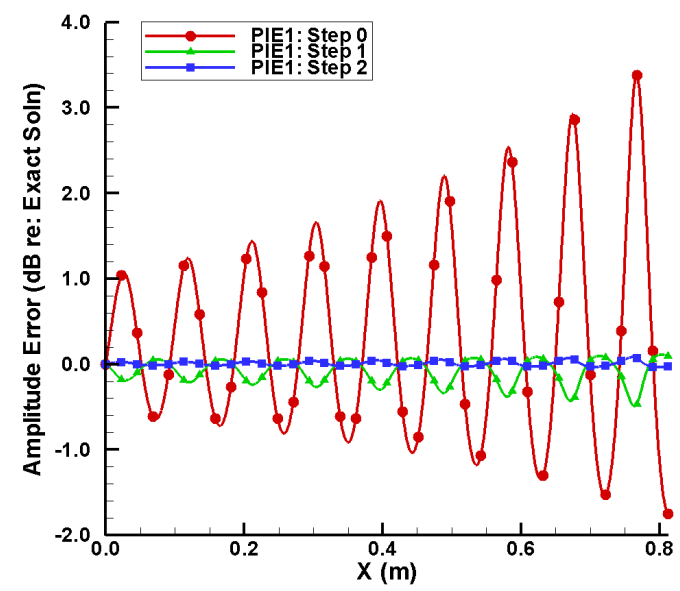

(a) Amplitude Error

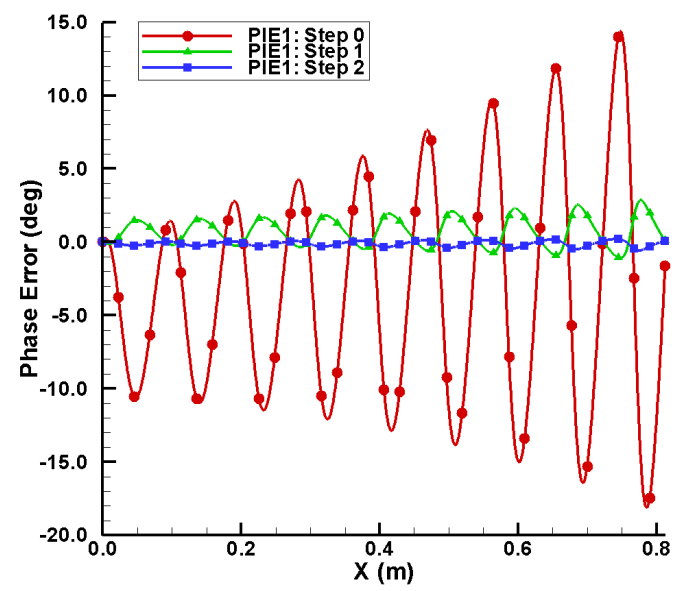

(b) Phase Error

Figure 10. Amplitude and phase error for several PIE1 iteration steps with reflections (least attenuated mode, treated duct: $n=1$, $\left.\zeta=(1.0,-0.5), \zeta_{\text {exit }}=(2.0,0.0), f=2000 \mathbf{H z}\right)$

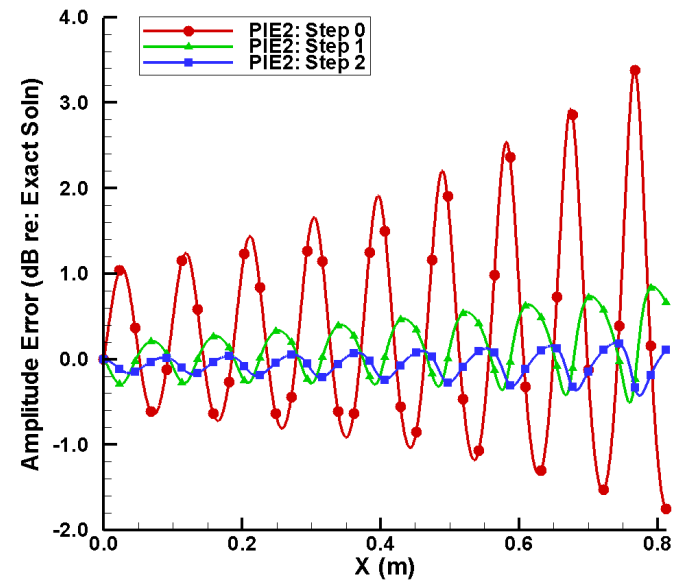

(a) Amplitude Error

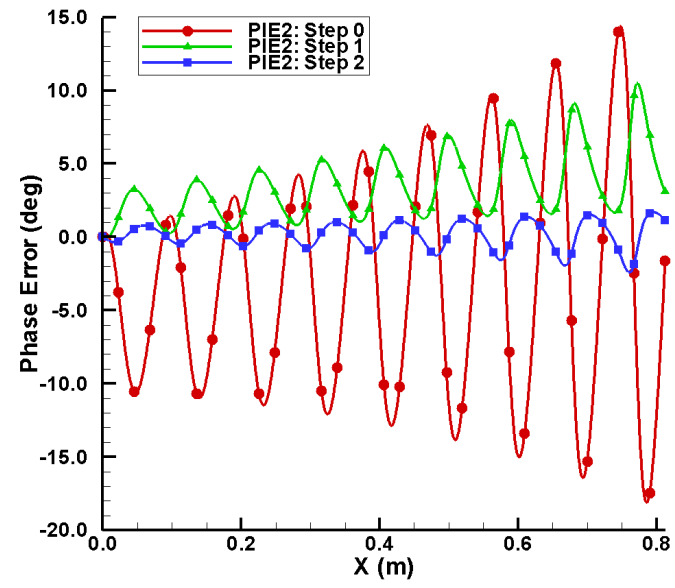

(b) Phase Error

Figure 11. Amplitude and phase error for several PIE2 iteration steps with reflections (least attenuated mode, treated duct: $n=1$, $\left.\zeta=(1.0,-0.5), \zeta_{\text {exit }}=(2.0,0.0), f=2000 \mathbf{H z}\right)$ 


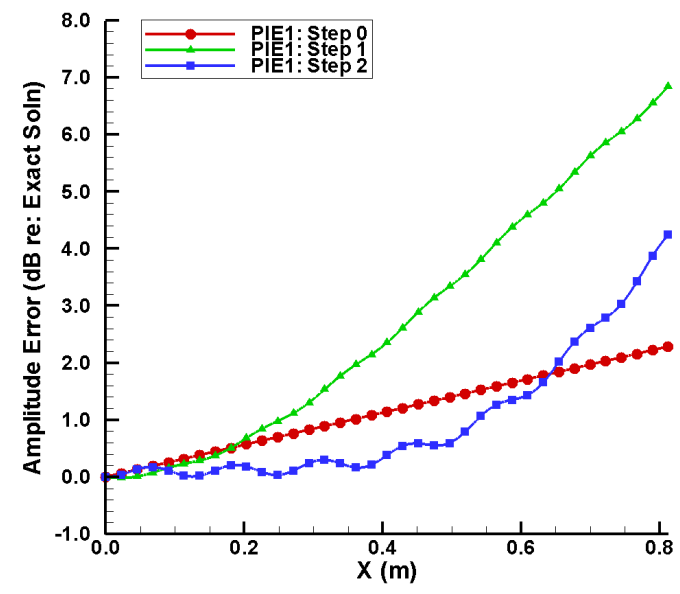

(a) Amplitude Error

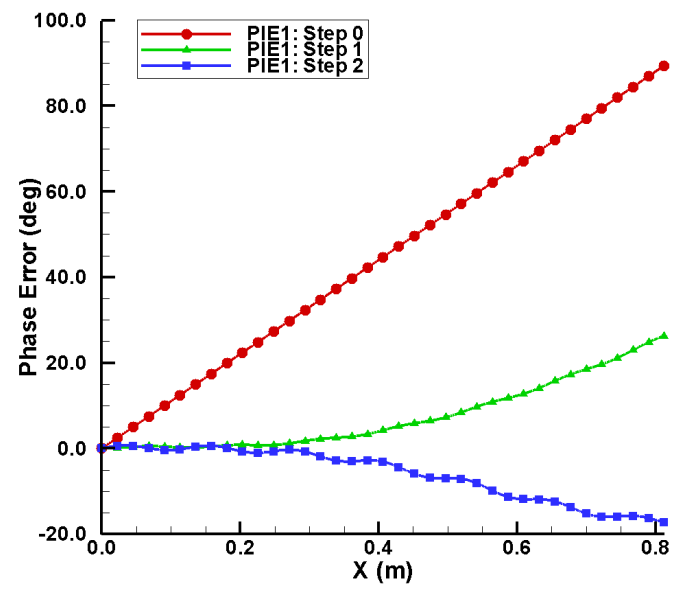

(b) Phase Error

Figure 12. Amplitude and phase error for several PIE1 iteration steps (least attenuated mode, treated duct: $n=3, \zeta=(1.0,-0.5)$, $f=2000 \mathbf{H z})$

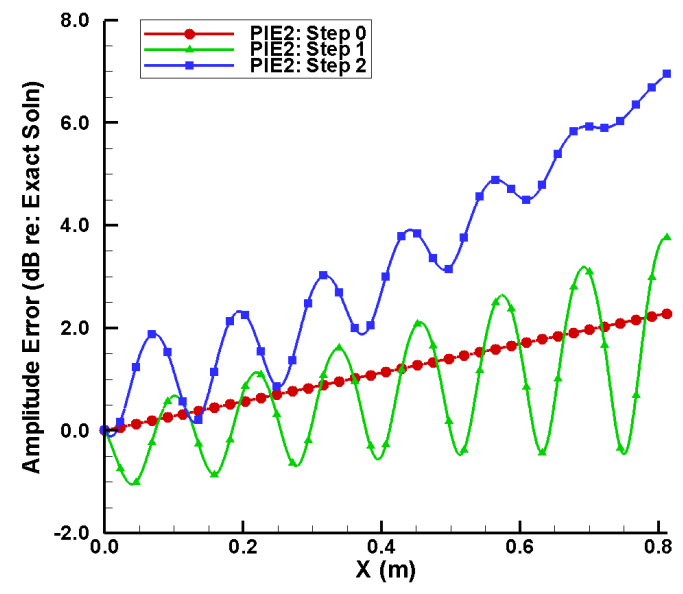

(a) Amplitude Error

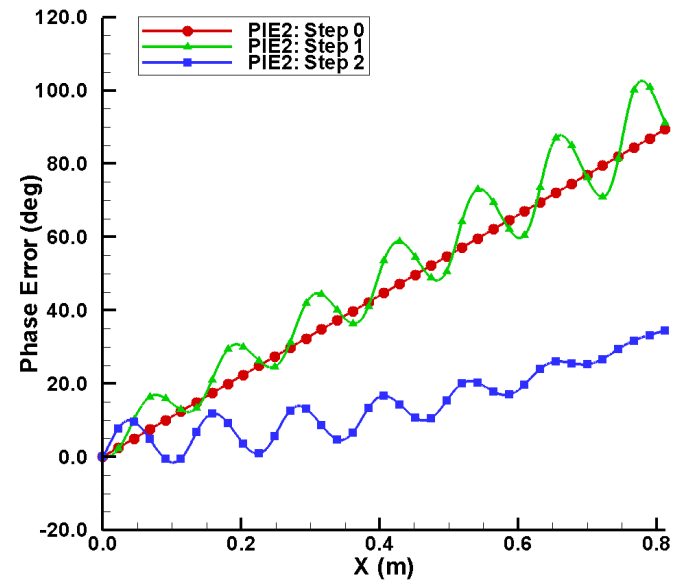

(b) Phase Error

Figure 13. Amplitude and phase error for several PIE2 iteration steps (least attenuated mode, treated duct: $n=3, \zeta=(1.0,-0.5)$, $f=2000 \mathbf{H z})$ 


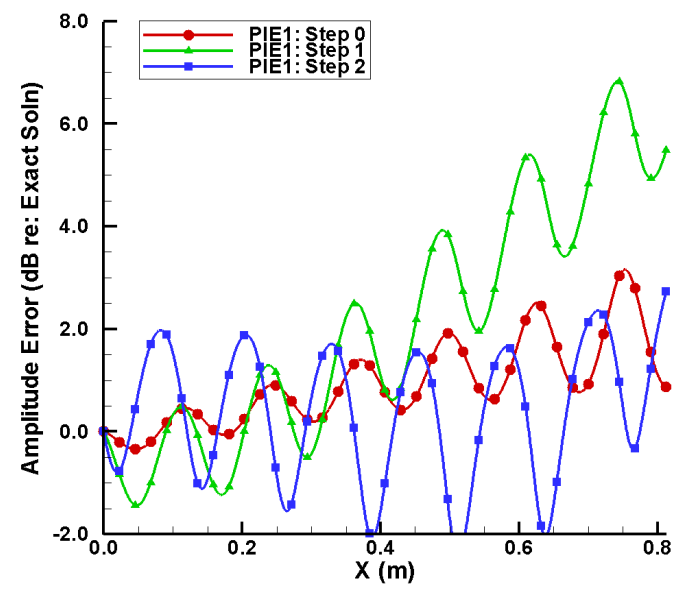

(a) Amplitude Error

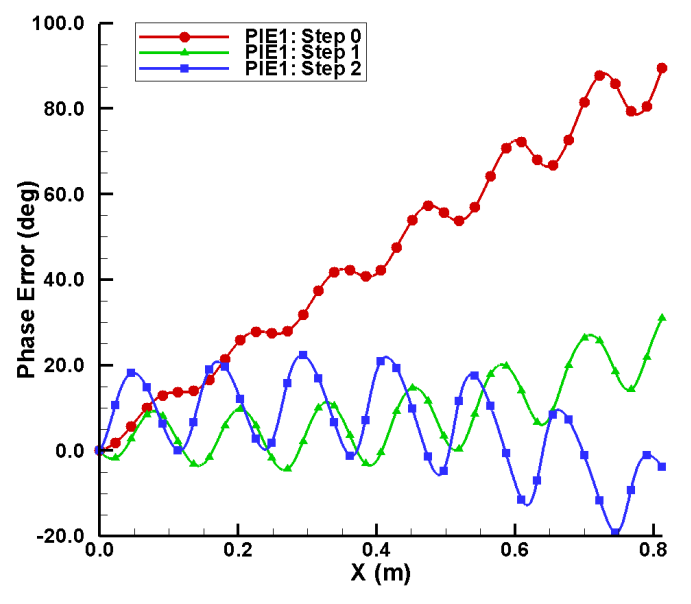

(b) Phase Error

Figure 14. Amplitude and phase error for several PIE1 iteration steps with reflections (least attenuated mode, treated duct: $n=3$, $\left.\zeta=(1.0,-0.5), \zeta_{\text {exit }}=(2.0,0.0), f=2000 \mathbf{H z}\right)$

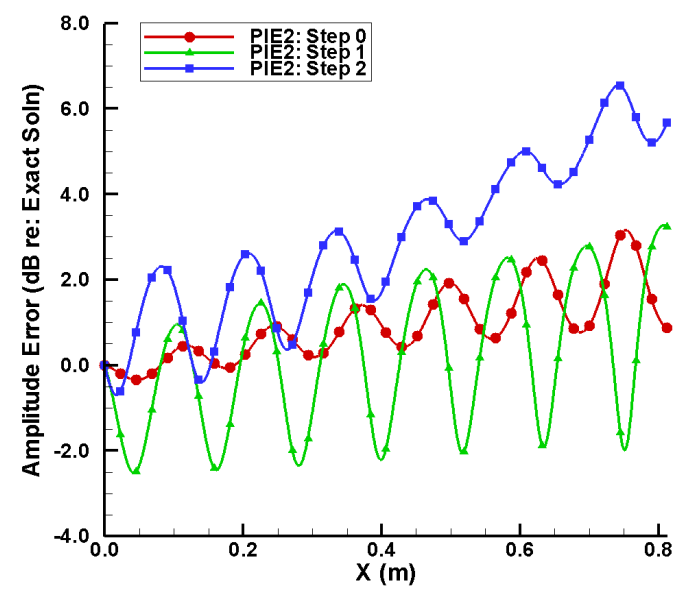

(a) Amplitude Error

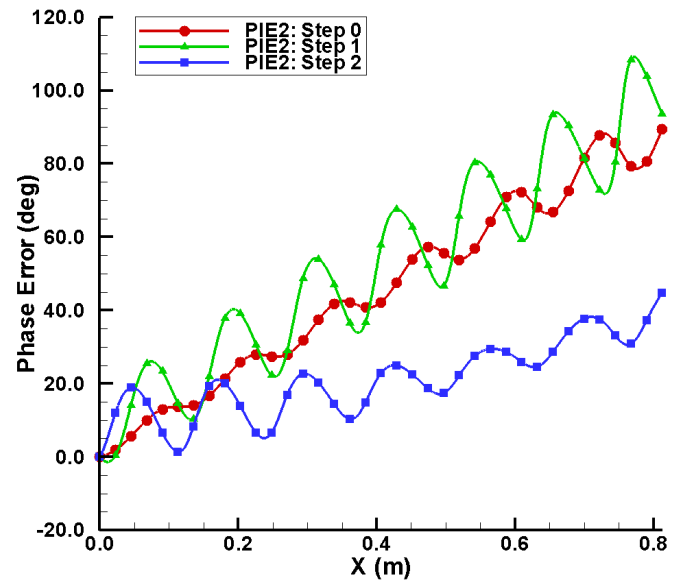

(b) Phase Error

Figure 15. Amplitude and phase error for several PIE2 iteration steps with reflections (least attenuated mode, treated duct: $n=3$, $\left.\zeta=(1.0,-0.5), \zeta_{\text {exit }}=(2.0,0.0), f=2000 \mathbf{H z}\right)$ 